\title{
Cisplatin plus paclitaxel and maintenance of bevacizumab on tumour progression, dissemination, and survival of ovarian carcinoma xenograft models
}

\author{
P Oliva ${ }^{1,5}$, A Decio ${ }^{1,5,6}$, V Castiglioni ${ }^{2}$, A Bassi ${ }^{3}$, E Pesenti ${ }^{4}$, M Cesca', E Scanziani ${ }^{2}$, D Belotti' and R Giavazzi ${ }^{*, 1}$ \\ 'Department of Oncology, Mario Negri Institute for Pharmacological Research, 20156 Milan, Italy; ${ }^{2}$ DIPAV, Faculty of Veterinary Medicine, University of \\ Milan, 20133 Milan, Italy; ${ }^{3}$ Dipartimento di Fisica, Politecnico di Milano, 20133 Milan, Italy; ${ }^{4}$ Nerviano Medical Sciences Oncology, 20014 Nerviano, Italy
}

BACKGROUND: Bevacizumab is being incorporated as first-line therapy with standard-of-care chemotherapy on epithelial ovarian carcinoma (EOC). We investigated bevacizumab combined with chemotherapy on tumour progression and mouse survival in EOC xenograft models.

METHODS: Bevacizumab was administered concomitantly with cisplatin plus paclitaxel (DDP + PTX), continued after induction (maintenance) or started after chemotherapy. The effect on tumour progression was monitored by bioluminescence imaging (BLI) ( IA9-luc xenograft). Tumour dissemination into the peritoneal organs and ascites formation (HOC22 xenograft) was evaluated by histological analysis at the end of treatment (interim) and at euthanasia (survival). The effects on overall survival (OS) were investigated in both EOC models.

RESULTS: Bevacizumab with PTX + DDP delayed tumour progression in mice bearing EOC xenografts. OS was significantly extended, with complete responses, by bevacizumab continued after stopping chemotherapy in the HOC22 xenograft. Bevacizumab alone inhibited ascites formation, with only limited effect on tumour burden, but combined with PTX + DDP reduced ascites and metastases. Bevacizumab started after induction with PTX + DDP and maintained was equally effective on tumour progression and survival on I A9-luc xenograft.

CONCLUSION: Bevacizumab combined with chemotherapy not only affected tumour progression, but when administered as maintenance regimen significantly prolonged survival, reducing ascites, and tumour dissemination. We believe our findings are consistent with the clinical results and shed light on the potential effects of this kind of treatment on tumour progression.

British Journal of Cancer (2012) I 07, 360-369. doi:I0.1038/bjc.2012.26I www.bjcancer.com

Published online 19 June 2012

(c) 2012 Cancer Research UK

Keywords: cisplatin; paclitaxel; bevacizumab; ovarian carcinoma; metastasis

Epithelial ovarian cancer (EOC) is a frequent cause of death in women in Western countries (Siegel et al, 2011). Cytoreductive surgery followed by platinum-based chemotherapy, usually combined with paclitaxel (PTX), is the standard first-line therapy (Cannistra et al, 2003). Despite an initial response in a high percentage of cases, most patients ultimately relapse with chemo-resistant disease.

Epithelial ovarian cancer causes widespread intraperitoneal carcinomatosis and voluminous ascites. Vascular endothelial growth factor (VEGF), originally described as a vascular permeability factor (Dvorak et al, 1991), is the main regulator of vascular proliferation and has a primary role in angiogenesis in different types of cancer (Ferrara, 2004). Vascular endothelial growth factor overexpression is frequently described in ovarian cancer patients and is associated with disease progression and with a worse outcome (Bamberger and Perrett, 2002). Ovarian cancers secrete large amounts of VEGF (Santin et al, 1999) and VEGF levels can

\footnotetext{
*Correspondence: Dr R Giavazzi; E-mail: raffaella.giavazzi@marionegri.it

${ }^{5}$ These authors contributed equally to this work.

${ }^{6}$ Fellow of 'Amiche del Mario Negri', Milan, Italy.

Received 5 March 2012; revised 15 May 2012; accepted 16 May 2012; published online 19 June 2012
}

serve as an additional prognostic factor (Kraft et al, 1999; Manenti et al, 2003). Vascular endothelial growth factor causes ascites to accumulate by increasing the permeability of diaphragmatic and tumour-associated vasculature (Barton et al, 1997). It is also an important autocrine growth factor in this disease (Boocock et al, 1995; Sher et al, 2009). Vascular endothelial growth factor is therefore an important therapeutic target for ovarian cancer.

Bevacizumab (Bev, Avastin), is a recombinant humanised monoclonal antibody directed against VEGF (Presta et al, 1997; Lin et al, 1999) that binds to and neutralises the biological properties of human VEGF (Kim et al, 1992) by blocking the interaction of VEGF with its receptor. Bevacizumab is approved for the treatment of metastatic colorectal (Hurwitz et al, 2004), non-small cell lung (Sandler et al, 2006), and renal cell cancer (Rini et al, 2008), in combination with standard therapy and as a single agent in recurrent glioblastoma multiforme (Cohen et al, 2009).

Preclinical studies tested the activity of bevacizumab on human EOC transplanted in mice and showed delayed tumour growth, reduction of ascites in the peritoneal cavity and improved overall survival (OS) (Hu et al, 2002; Gerber and Ferrara, 2005; Mabuchi et al, 2008). In those studies, the maximal benefit of VEGF blockade was observed in combination with chemotherapy. 
Phase II trials evaluated bevacizumab on recurrent platinumresistant EOC and, unlike in other solid tumours, it was active as monotherapy (Burger et al, 2007; Cannistra et al, 2007). Several clinical studies have combined bevacizumab with carboplatin, PTX, or other biologic and cytotoxic agents in advanced ovarian cancer (Chura et al, 2007; Nimeiri et al, 2008; O'Malley et al, 2011). Two multi-centric phase III studies, the American GOG218 and the international ICON7, have tested bevacizumab as front-line therapy with carboplatin and PTX in patients with advanced EOC. The results on the primary end point, progression-free survival (PFS), were published while this manuscript was being finalised. GOG218 found significant improvement in PFS in patients randomised to the regimen with bevacizumab plus chemotherapy followed by extended bevacizumab (Bev-throughout) (Burger et al, 2011). Progression-free survival was also prolonged in the arm with bevacizumab added to chemotherapy and extended after chemotherapy in the ICON7 study (Perren et al, 2011). These data formed the basis for the recent approval in Europe of bevacizumab in women with advanced ovarian cancer.

In this study with preclinical models of EOC, we investigated some still open questions on the use of bevacizumab in combination with chemotherapy. To address the importance of maintenance therapy with bevacizumab added to first-line chemotherapy (PTX plus a platinum-based drug), we tested whether cisplatin (DDP)/PTX as mono- or combined treatment, plus bevacizumab affected tumour progression and whether bevacizumab administered in a maintenance regimen prolonged the survival of mice. Furthermore, we tested whether the responsiveness of the tumour to chemotherapy influence the response to the combination with bevacizumab and whether the addition of bevacizumab, after a clinical response to PTX + DDP, prolonged the survival of the mice. The second aim of the study was to analyse the contributions of chemotherapy (PTX + DDP) and inhibitors of angiogenesis (Bev) in controlling tumour spread and progression to secondary organs, and ascites formation in the peritoneal cavity of mice.

\section{MATERIALS AND METHODS}

\section{Drug preparations}

Paclitaxel (Indena S.p.A., Milan, Italy) was dissolved in 50\% Cremophor EL (Sigma-Aldrich, Milan, Italy) and 50\% ethanol and further diluted with saline before use. Cisplatin (DDP, SigmaAldrich) was dissolved in $0.9 \% \mathrm{NaCl}$. Bevacizumab (Bev, Avastin, Roche S.p.A., Milan, Italy) was diluted in $0.9 \% \mathrm{NaCl}$ before use.

\section{Animals}

Six- to eight-week-old female NCr-nu/nu mice were obtained from Harlan (Correzzana, Italy). They were maintained under specific pathogen-free conditions, housed in isolated vented cages, and handled using aseptic procedures. Procedures involving animals and their care were conducted in conformity with institutional guidelines that comply with national (Legislative Decree 116 of 27 January 1992, Authorization n.19/2008-A issued 6 March 2008, by the Italian Ministry of Health) and international laws and policies (EEC Council Directive 86/609, OJ L 358, 1, 12 December 1987; standards for the Care and Use of Laboratory Animals, United States National Research Council, Statement of Compliance A5023-01, 28 October 2008), and in line with Guidelines for the welfare and use of animals in cancer research (Workman et al, 2010).

\section{Human ovarian carcinoma models}

HOC22 xenograft is a high-grade serous carcinoma derived from a patient with malignant ascites (Massazza et al, 1989) and transplanted as a $10 \times 10^{6}$ ascites-derived cell suspension into the peritoneal cavity of nude mice. This xenograft was molecularly, biologically, and pharmacolgically characterised and stored as frozen stock at early passage after establishment from patient (our working stock) (Nicoletti et al, 1993; Codegoni et al, 1998; Garofalo et al, 2003). This xenograft was selected for testing bevacizumab in combination with chemotherapy from a panel of EOC-derived xenografts on the basis of its sensibility to PTX and DDP single treatments.

1A9-luc is a variant derived from the A2780-1A9 human ovarian carcinoma cell line (kindly provided by Tito Fojo, NCI-NIH, Bethesda, MD, USA) infected with lentiviral vector carrying the coding sequence of synthetic firefly luciferase gene, luc2 (Photinus pyralis). 1A9-VS1 is its highly angiogenic cell variant stably transfected with VEGF(121), that forms ascites and high level of plasma VEGF (Manenti et al, 2005). These cell lines were molecularly and pharmacologically characterised (Giannakakou et al, 2000; Bani et al, 2004; Manenti et al, 2005) and their DNA fingerprint (short tandem repeat profiling, AmpFISTR Identifiler Plus PCR Amplification kit, Applied Biosystems, Monza, Italy) found similar to the A2780. Stocks of the cell line were stored frozen in liquid nitrogen and kept in culture for no more than 8 weeks before injection in mice. 1A9-luc and 1A9-VS1 were maintained in RPMI 1640 (Voden Medical Instrument S.p.A., Meda, Italy) supplemented with 10\% FCS (Lonza Sales, Basel, Swizerland) and implanted into the peritoneal cavity of nude mice as a $10 \times 10^{6}$ cell suspension (Manenti et al, 2005).

\section{Preclinical trials}

Tumour-bearing mice were randomised (by histological analysis for HOC22; by BLI for 1A9-luc) after tumour transplant (10-15 mice in each group). Mice were treated with PTX and DDP, singly or together (PTX + DDP). Doses and schedules of chemotherapy were selected from previous tests that gave a minimal/ moderate response as single treatment (data not shown). Bevacizumab was administered according to three protocols: (a) in combination with chemotherapy, (b) in combination with chemotherapy and continued after the end of the therapy, (c) starting $24 \mathrm{~h}$ after the end of the chemotherapy. In the combination studies, chemotherapy was administered $24 \mathrm{~h}$ before bevacizumab. Detailed schedules, doses and lengths of treatment are reported in the Results (Figures 1A, 3A, 4A and 5A). Vehicles were administered at the same schedule and route as the active compounds.

\section{Treatment evaluation}

For HOC22-bearing mice, tumour dissemination was analysed histopathologically while in 1A9-luc-bearing mice tumour burden was monitored by BLI. Survival time and toxicity were recorded for all the mice (see Supplementary Methods for detailed analysis).

\section{ELISA}

Peripheral venous blood samples were collected in $3.8 \%$ trisodium citrate dihidrate and immediately centrifuged at 3000 r.p.m. for $20 \mathrm{~min}$. Plasma was aliquoted and stored at $-80^{\circ} \mathrm{C}$ until processing. Human VEGF was measured by an ELISA (Quantikine Human VEGF Immunoassay; R\&D Systems, Milan, Italy) according to the manufacturer's directions, as described previously (Manenti et al, 2005).

\section{Statistical analyses}

Statistical analyses were done using Prism Software (Prism 5.01; GraphPad Software, La Jolla, CA, USA). Differences in survival times (at killing) were analysed by the Log-Rank test. Differences in photon counts were analysed by two-way ANOVA followed by the Bonferroni post-test. Differences in histological scores were 
A
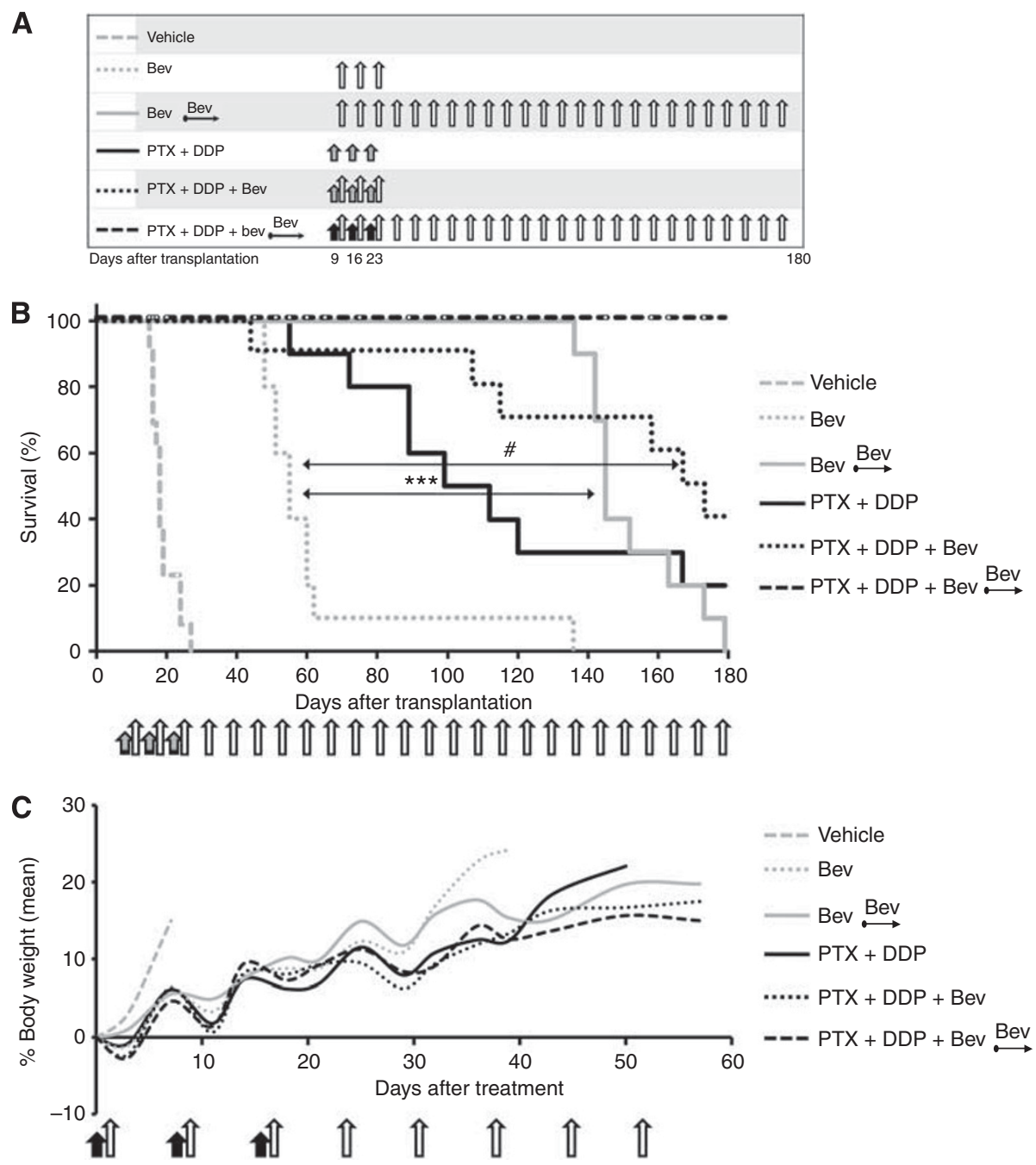

Figure I Antitumour activity of DDP + PTX combined with bevacizumab on HOC22 xenografts. (A) Protocol of the study: treatments started 9 days after HOC22 transplanted intraperitoneally in nude mice. PTX $\left(10 \mathrm{mg} \mathrm{kg}^{-1}\right)+$ DDP $\left(2.5 \mathrm{mg} \mathrm{kg}^{-1}\right)$, and Bev $\left(5 \mathrm{mg} \mathrm{kg}^{-1}\right)$ alone or in combination with chemotherapy (PTX + DDP + Bev), were injected i.v. every 7 days. Bevacizumab was stopped after three chemotherapy cycles or continued for another 22 cycles (Bev). (B) Effect of PTX + DDP, Bev alone or in combination on the lifespan of mice bearing HOC22 $\left(n=10,{ }^{\sharp} P=0.0004,{ }^{*} * * * P=0.004\right)$.

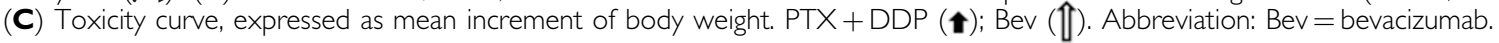

calculated by one-way ANOVA followed by Dunn's multiple comparison test.

\section{RESULTS}

\section{Cisplatin plus paclitaxel and bevacizumab induction $v s$ maintenance regimen in a patient-derived ovarian carcinoma xenograft model}

PTX + DDP combined with bevacizumab were tested on HOC22 xenograft model derived from a patient's EOC and transplanted in the peritoneal cavity of nude mice. Nude mice bearing HOC22 were randomised (10 mice per group) on day 9, on the basis of representative mice with confirmed presence of tumour in the peritoneal cavity (advanced stage tumour), and treated with PTX + DDP and bevacizumab in combination regimens. Doses and schedules of the trial are described in Figure 1A.

At the doses and schedules used, PTX and DDP single agents were not efficacious, whereas the addition of bevacizumab significantly prolonged survival for both combinations (Supple- mentary Data, Table 1). Figure 1B shows that PTX + DDP significantly increased survival (MST 106 days, ILS 489\%). The survival was further increased with the addition of bevacizumab to the chemotherapy (MST 170 days, ILS $844 \%$, 4 out of 10 tumourfree mice). Maintenance treatment with bevacizumab alone inhibited tumour progression and significantly prolonged survival compared with bevacizumab interrupted after 3 weeks (MST 145 days, ILS $706 \%$ and MST 55 days, ILS 206\%). Only the combination of the two chemotherapeutics with bevacizumab in maintenance treatment $(\mathrm{PTX}+\mathrm{DDP}+\mathrm{Bev} \rightarrow \mathrm{Bev} ; 22$ weeks after the 3 weeks of induction) resulted in tumour-free mice. After stopping bevacizumab (day 180) 4 out of 10 mice eventually developed tumours (day 188, 233, 331 and 335), whereas absence of tumour was confirmed in the remaining mice at necroscopy (complete response).

The triple combination (DDP $+\mathrm{PTX}+\mathrm{Bev})$ and maintenance regimens (PTX $+\mathrm{DDP}+\mathrm{Bev} \rightarrow \mathrm{Bev}$ ) were well tolerated, with no clinical signs of toxicity throughout the study or significant body weight loss (Figure 1C). These trials indicate an advantage adding bevacizumab to chemotherapy, which was amplified by a maintenance regimen with bevacizumab after chemotherapy. 
Effect of chemotherapy combined with bevacizumab on intra-abdominal dissemination and ascites formation

Dissemination into the peritoneal cavity is the primary route of progression in ovarian cancer, and ascites and tumour burden correlate with prognosis. HOC22 produces ascites and disseminates in the organs of the peritoneal cavity of nude mice, so biological parameters modelling the human disease can be studied (Massazza et al, 1989; Garofalo et al, 2003). We examined the effect of bevacizumab alone or combined with chemotherapy on HOC22 dissemination at the end of induction treatment (interim analysis, day 24, except for vehicle-treated mice which were euthanised on day 18 when signs of discomfort were noticed) and at killing. Necroscopy on representative mice (4-6 per group) showed macroscopical reduction of ascites, but more HOC22 neoplastic aggregates in mice treated with bevacizumab alone both at interim and killing; reduction of tumour masses was observed after chemotherapy (Figure 2A).
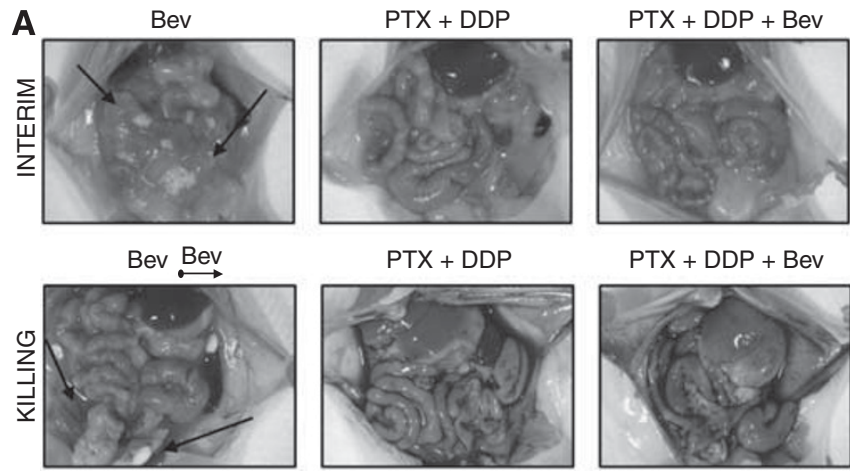

$\mathrm{PTX}+\mathrm{DDP}$

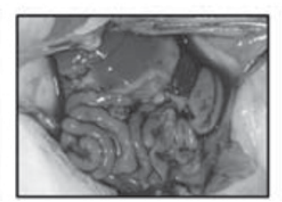

$\mathrm{PTX}+\mathrm{DDP}+\mathrm{Bev}$

B
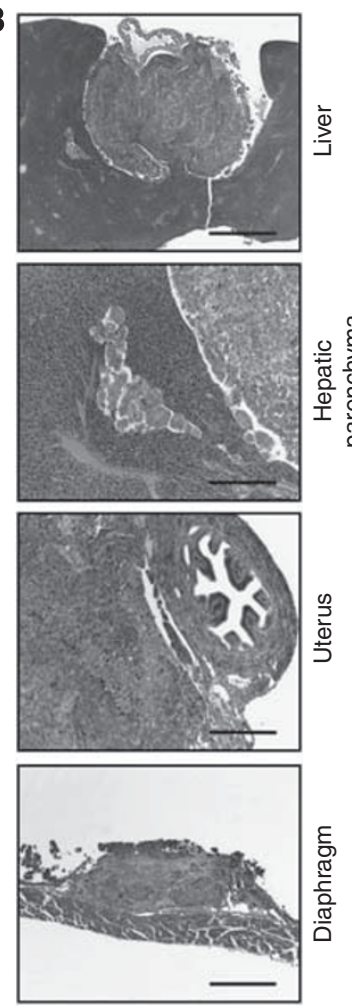

C
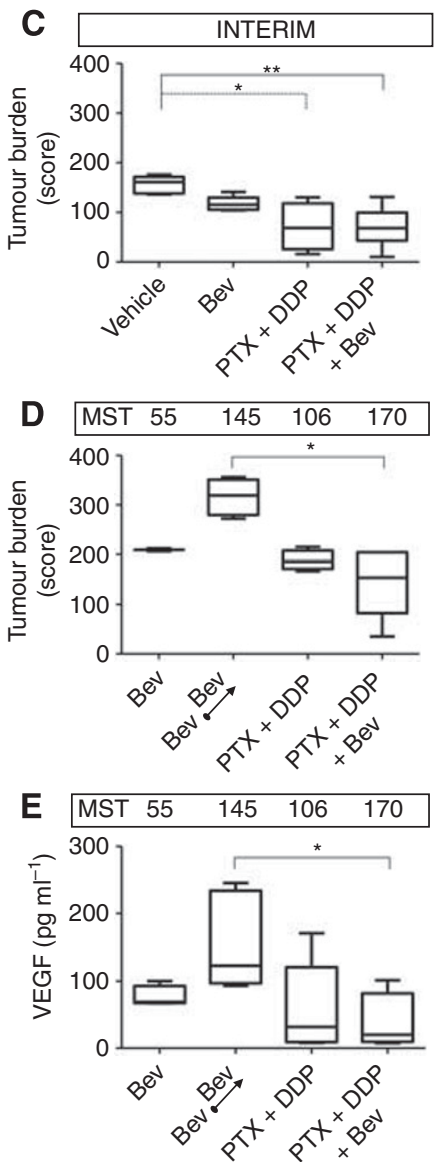

The liver, ovaries and uterus, diaphragm and pancreas are the organs most commonly involved in tumour dissemination (Figure 2B). Figures 2C and D report the histopathological scores (tumour burden) for each group at interim and killing (MST). At interim analysis, the vehicle group had the highest tumour burden and amount of ascites, and mice treated with PTX + DDP + Bev had the lowest. The interim analysis indicated that bevacizumab alone reduces ascites formation (undetectable), but does not limit tumour burden (Figure 2C). However, bevacizumab added to PTX + DDP (PTX + DDP + Bev) reduced both ascites and tumour burden (Figure 2C). The treatment with bevacizumab caused a significant decrease of microvessel density (data not shown), confirming the effect of bevacizumab on tumour vasculature. As expected, tumour scores at killing (Figure 2D) were higher than those at interim analysis, but with the same pattern. The tumour burden was highest in the bevacizumab maintenance group, with minimal ascites (not measurable) and a wider range of dissemination than in the other groups (Figure 2D). The difference in plasma VEGF measured at killing in the same mice matched the tumour burden (Figures 2D and E).

Necropsy on the four mice that developed HOC22 after the suspension of bevacizumab maintenance (PTX $+\mathrm{DDP}+\mathrm{Bev} \rightarrow$ Bev) showed abundant ascites, with diffuse carcinomatosis and involvment of the peritoneal organs (data not shown).

These findings indicate the combination of chemotherapy and bevacizumab are relevant to resolve tumour burden and ascites progression.

\section{The response to bevacizumab combined with chemotherapy is related to the drug sensitivity of the ovarian carcinoma xenograft}

The effect of bevacizumab added to PTX or DDP, single agents, was investigated in the 1A9-luc model that serves to follow tumour progression with BLI and shows a different profile of sensitivity to PTX (responsive, Figure 3) and DDP (moderately responsive Figure 4). Mice transplanted with 1A9-luc were randomised on day 6 with the aid of BLI on the basis of photon counts $(10$ mice per group). PTX (trial of Figure 3) or DDP (trial of Figure 4) and bevacizumab were administered singly or in combination, with bevacizumab administered concomitant to chemotherapy (for three cycles) or continued for additional 14 cycles after its suspension (maintenance). Doses and schedules of the trials are

Figure 2 Effect of bevacizumab with chemotherapy on tumour burden, ascites formation and production of VEGF in mice bearing HOC22. Mice bearing HOC22 were treated with PTX + DDP combined with Bev (as described in Figure I) then necropsied at the end of induction treatment (interim) or at killing. (A) Exemplificative macroscopic figures for each group of mice bearing HOC22 at interim and killing. Grossly, HOC22 dissemination in the peritoneal cavity was characterised by whitish nodules scattered throughout the serosal surface of the organs. Note that Bev (interim) and Bev $\rightarrow$ Bev (killing)-treated mice presented the highest quantity of masses (black arrows) with respect to the other groups. (B) Representative histological images of the organs involved from HOC22-bearing mice. Note the neoplastic mass in the mesentery between the liver and the gallbladder, which is the most common hepatic site of dissemination (HE, Bar $=500 \mu \mathrm{m}$ ). Close-up of the mass in the hepatic parenchyma with neoplastic cells arranged in cords and lobules, with scant fibrovascular stroma (HE, Bar $=125 \mu \mathrm{m})$. The uterus is almost completely effaced by metastatic growth, with intralesional areas of necrosis and haemorrhage $(H E, B a r=125 \mu \mathrm{m})$. Massive metastatic invasion of the diaphragm, with neoplastic cells in several layers (HE, Bar $=250 \mu \mathrm{m})$. (C and $\mathbf{D})$ Quantification of intra-abdominal dissemination at interim (C) and at killing (D). Tumour burden in the peritoneal organs examined and quantified by a histological score $(n=4-6, * * P<0,01$, $* P<0,05)$. (E) Plasma VEGF levels measured at killing $(n=5, * P<0,05)$. Abbreviations: Bev = bevacizumab; MST = median survival time (killing). 
A

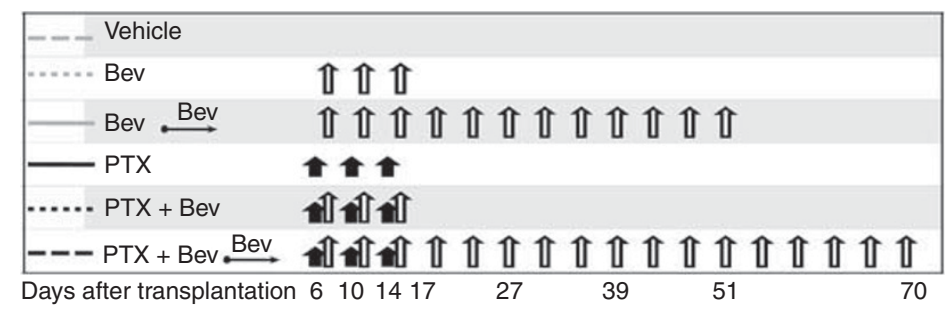

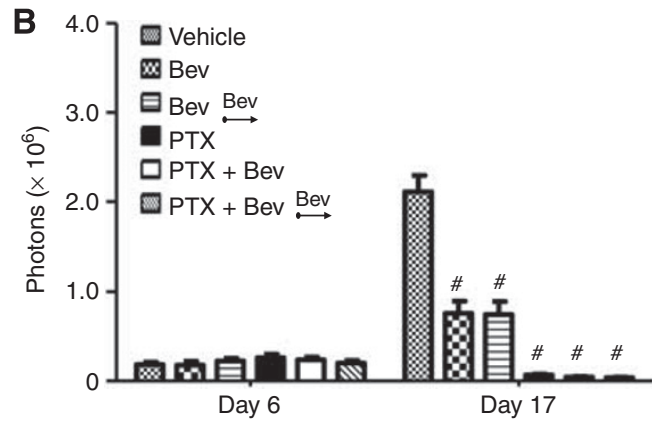

Days after transplantation

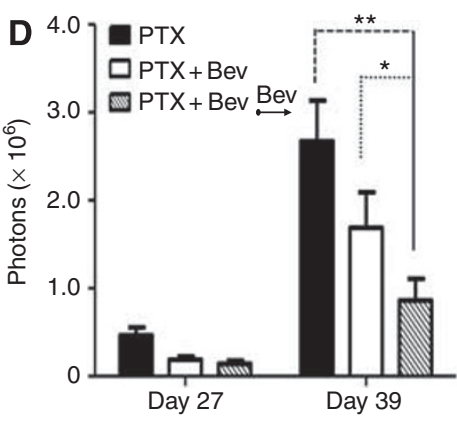

Days after transplantation
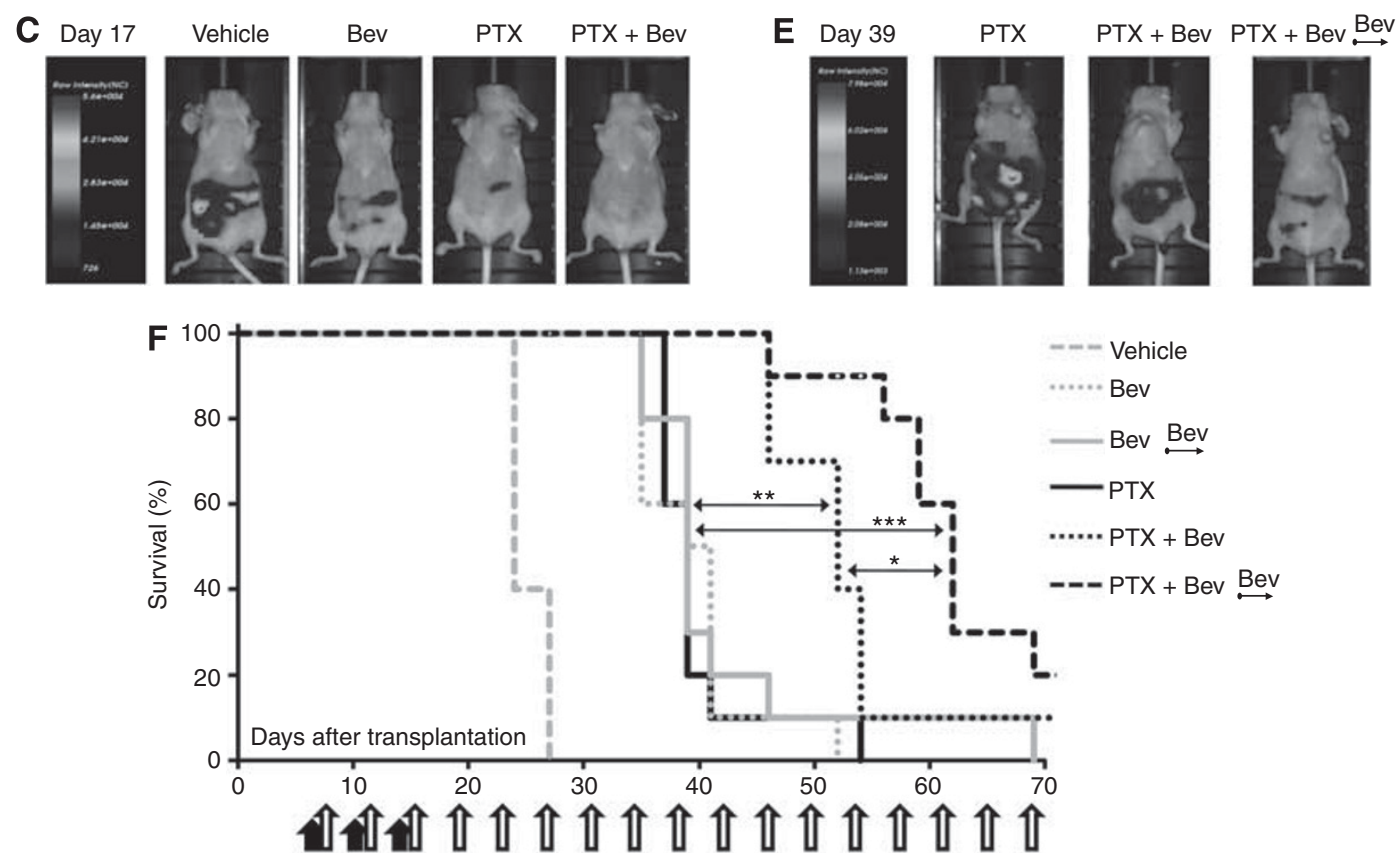

Figure 3 Antitumour activity of PTX in combination with bevacizumab on IA9-luc ovarian carcinoma xenograft. (A) Protocol of the study: treatments started 6 days after intraperitoneal I A9-luc transplantation in nude mice and were given every 4 days. PTX (-) was used at the dose of 20 mg kg ${ }^{-1}$, i.V., Q4 $\times 3$; Bev ( $\Uparrow, 5 \mathrm{mg} \mathrm{kg}^{-1}$, i.p.) was interrupted after three cycles of chemotherapy or continued till death (Bev). (B and D) Mice were monitored by BLI on day 6 (randomisation) and days 17,27, 39 (tumour progression) and the tumour burden was expressed as photon counts ( $n=\mid 0,{ }^{\#} P<0.001$ vs vehicle ${ }^{*} * \mathrm{P}<0.00 \mathrm{I}$ vs PTX and $* P<0.01$ vs PTX $\left.+\mathrm{Bev}\right)$. ( C and $\left.\mathbf{E}\right)$ One representative mouse treated with vehicle, Bev, PTX, PTX + Bev or PTX + Bev $\rightarrow$ Bev imaged $(\mathbf{C})$ on day 17 and $(\mathbf{E})$ on day 39. (F) Effect of the different treatments on the lifespan of the mice $(n=10$; $* * * P<0.000 I$, *** $P=0.01$, *P=0.02). Abbreviation: Bev = bevacizumab.

described in Figures 3A and 4A. Detailed results are in Supplementary Data, Table 2 and Table 3.

Paclitaxel delayed the progression of 1A9-luc (Figure 3). At the end of the three treatment cycles (day 17, Figures $3 \mathrm{~B}$ and $\mathrm{C}$ ) photon counts were lower in mice treated with PTX alone or combined with bevacizumab than in those given bevacizumab alone. The effect of bevacizumab in combination with PTX was evident at day 27 and even more at day 39 (Figures 3D and E); the number of photons from mice given bevacizumab after stopping PTX (PTX $+\mathrm{Bev} \rightarrow \mathrm{Bev})$ was significantly lower than in mice treated with bevacizumab concomitantly with PTX but for only three cycles (PTX + Bev). Survival reflected the progression of the tumour in the peritoneal cavity (Figure 3F). Paclitaxel and bevacizumab prolonged survival (MST 39 days, ILS 63\% and MST 40 days, ILS 67\%, respectively; Supplementary data, Table 2), but not survival advantage was observed with bevacizumab as single agent continued beyond the three cycles of treatment (Bev $\rightarrow \mathrm{Bev} v s \mathrm{Bev})$. However, the addition of bevacizumab to PTX significantly improved survival (MST 52 days, ILS 117\%) and continuing bevacizumab after PTX further improved survival (MST 62 days, ILS 158\%). 

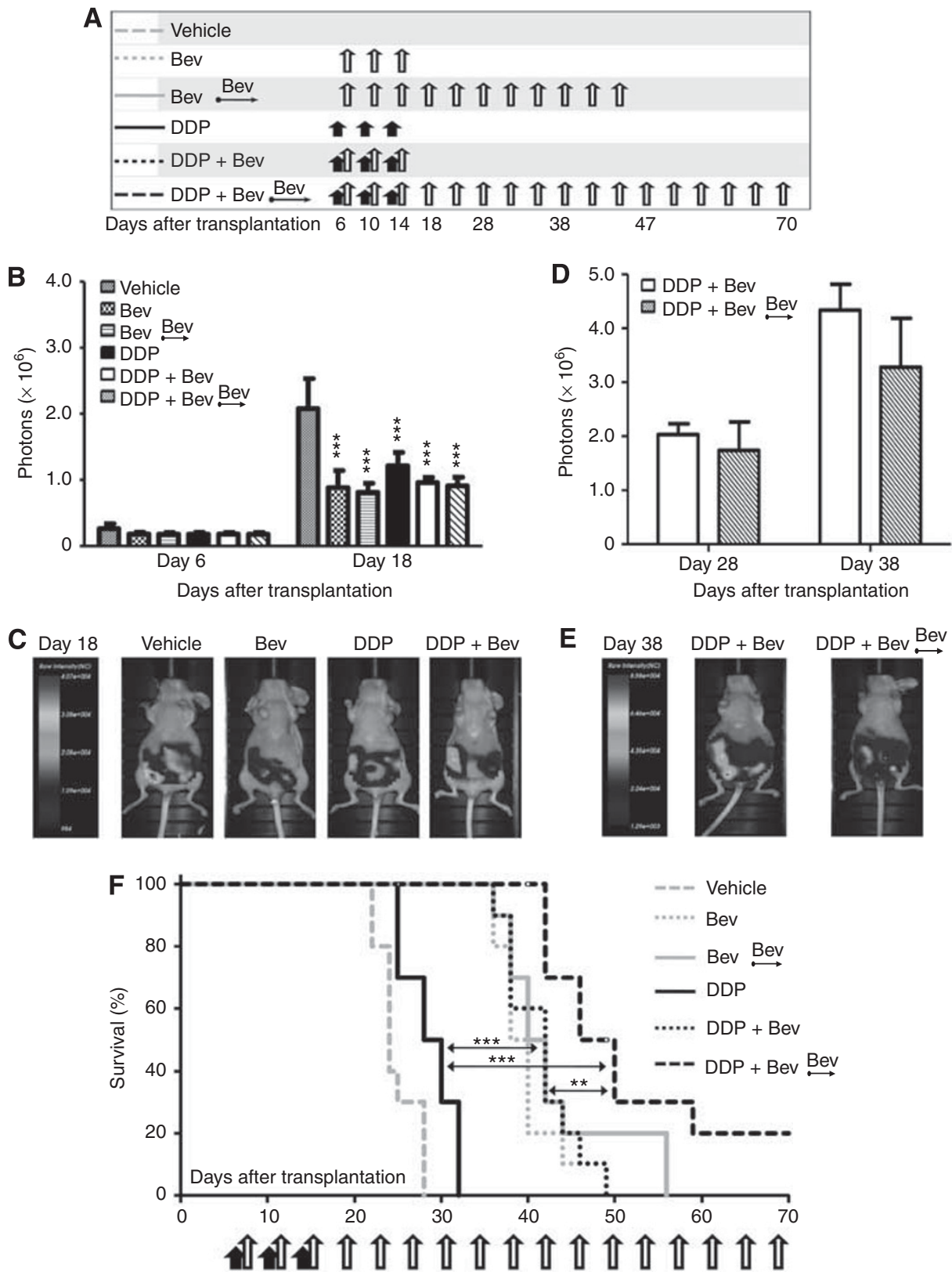

Figure 4 Antitumour activity of DDP in combination with bevacizumab on IA9-luc ovarian carcinoma xenograft. (A) Protocol of the study: DDP (-) started 6 days after tumour transplantation, at $4 \mathrm{mg} \mathrm{kg}^{-1}$ i.v, $\mathrm{Q} 4 \times 3$ and $\mathrm{Bev}$ ( $\Uparrow, 5 \mathrm{mg} \mathrm{kg}^{-1}$, i.p.) was stopped after three cycles, or continued every 4 days until death $(\stackrel{B e v}{\rightarrow})$. (B, D) Mice were imaged on day 6 and days 18, 28, 38 and tumour burden was expressed as photon counts ( $n=10$, **** $P<0.00$ I vs vehicle). (C and E) One representative mouse treated with vehicle, Bev, DDP, DDP + Bev, or DDP $+B e v \rightarrow B e v$ imaged $(\mathbf{C})$ on day I 8 and $(\mathbf{E})$ on day 38. (F) Effect of the treatments on the lifespan of mice bearing IA9-luc $(n=10, * * * P<0.000$ I vs DDP, ** $P<0.05$ vs DDP + Bev). Abbreviation: $\mathrm{Bev}=$ bevacizumab.

Photon counts indicated delay of tumour progression (day 18, Figures $4 \mathrm{~B}$ and $\mathrm{C}$ ) in mice treated with bevacizumab or DDP, but no significant advantage on combining the two (DDP $+\mathrm{Bev}$ ) (Figures 4B and C). Continuing bevacizumab after chemotherapy (DDP $+\mathrm{Bev} \rightarrow$ $\mathrm{Bev}$ ) affected the tumour progression (Figures $4 \mathrm{D}$ and $\mathrm{E}$ ), though not significantly. However, this regimen too prolonged survival (MST 48 days, ILS 100\%), compared with DDP alone (MST 29 days, ILS 21\%), bevacizumab alone (MST 40 days, ILS 67\%) or DDP + Bev for only three cycles (MST 42 days, ILS 75\%) (Figure 4F).

These trials confirm an advantage adding bevacizumab to chemotherapy, but the magnitude of the response depended from the sensitivity of the tumour to chemotherapy.
Bevacizumab after induction with chemotherapy prolongs survival equally independently of VEGF production by the tumour

Having seen that bevacizumab added to chemotherapy inhibited intraperitoneal tumour dissemination and prolonged survival, we wondered whether it was useful to give bevacizumab concomitantly with chemotherapy. In the trial described in Figure 5 mice bearing 1A9-luc received PTX + DDP + Bev, continuing bevacizumab as maintenance (PTX $+\mathrm{DDP}+\mathrm{Bev} \rightarrow \mathrm{Bev}$, as above) or starting bevacizumab after induction with chemotherapy (PTX + $\mathrm{DDP} \rightarrow \mathrm{Bev}$ ) (Figure 5A). 


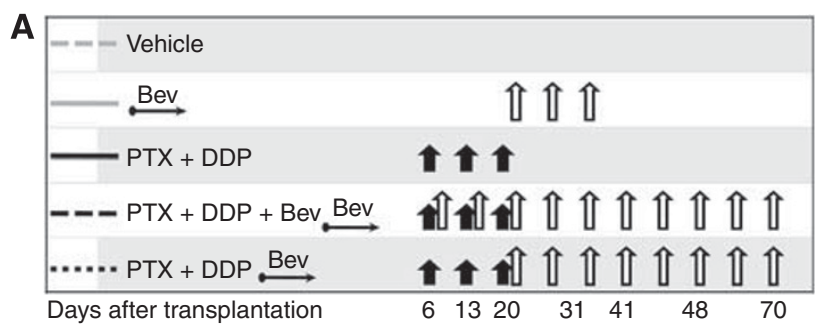

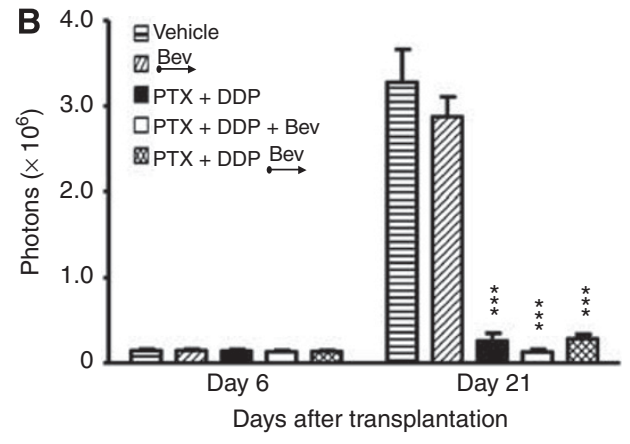

C

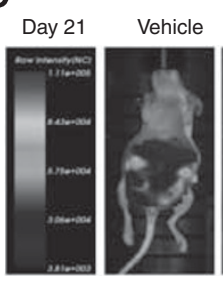

PTX+DDP+

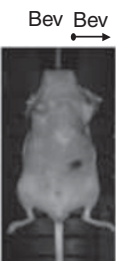

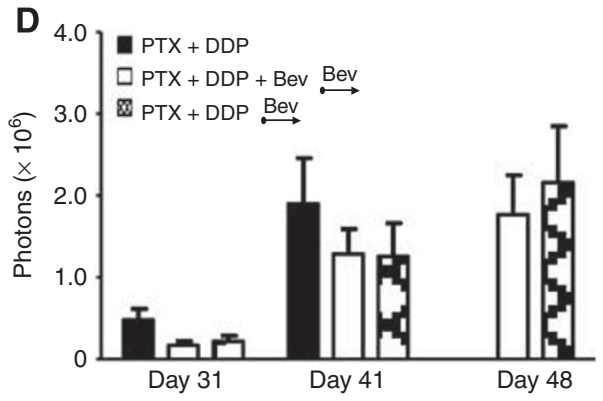

Days after transplantation

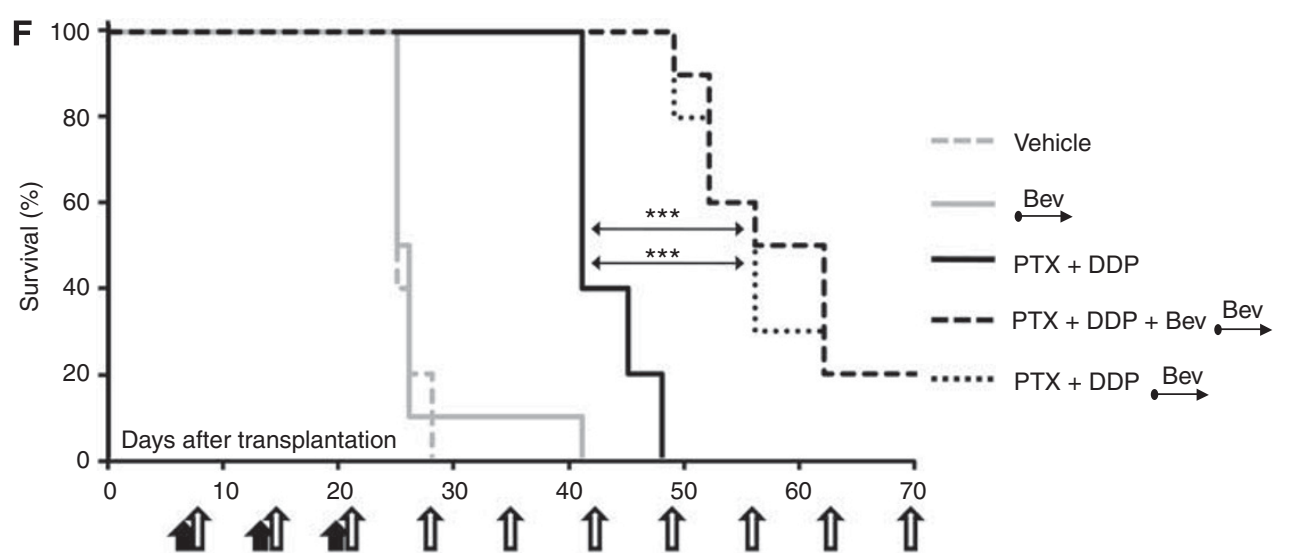

Figure 5 Antitumour activity of bevacizumab administered after induction with chemotherapy on I A9-luc ovarian carcinoma xenograft. (A) Protocol of the study: at randomisation (day 6, by BLI) mice received PTX $\left(20 \mathrm{mg} \mathrm{kg}^{-1}\right)+\operatorname{DDP}\left(4 \mathrm{mg} \mathrm{kg}^{-1}\right)$ i.v. every 7 days $(\mathbf{-})$. Bev $\left(5 \mathrm{mg} \mathrm{kg}{ }^{-9} \text {, i.p, } \Uparrow\right)^{2}$ was given in combination with PTX + DDP (PTX + DDP + Bev $\rightarrow \mathrm{Bev}$ ) or started $24 \mathrm{~h}$ after the end of the three cycles of chemotherapy (PTX + DDP $\rightarrow$ Bev) and continued weekly until death. (B and D) Mice were monitored by BLI on day 6 (randomisation) and days 2I, 3I, 4I, 48 (tumour progression) and tumour burden was expressed as photon counts $(n=10$, ****P $<0.001)$. (C and E) One representative mouse treated with vehicle, $\rightarrow$ Bev, PTX + DDP, PTX + DDP + Bev $\rightarrow$ Bev, or PTX + DDP $\rightarrow$ Bev imaged (C) on day $2 I$ and $(\mathbf{E})$ on day $4 I$. (F) Effects of the different treatments on the lifespan of mice bearing IA9-luc $(n=10$, **** $P<0.000 \mid)$. Abbreviation: Bev = bevacizumab.

By day 21 the tumour burden (expressed as photon counts) was equally inhibited in the PTX + DDP and PTX + DDP + Bev groups (Figures $5 \mathrm{~B}$ and $\mathrm{C}$ ). Bevacizumab alone started on day 21 had no effect on advanced tumour burden. The lack of difference between $\mathrm{PTX}+\mathrm{DDP}+\mathrm{Bev}$ followed by bevacizumab and PTX + DDP starting bevacizumab at the end of induction chemotherapy was evident on disease progression, evaluated as BLI analysis at days 31, 41, and 48 (Figures 5D and E). The group treated with PTX + DDP only had a higher photon count, though not significantly so, indicating that bevacizumab is necessary to control recurrence.
In fact, bevacizumab added to chemotherapy did significantly prolong the survival of the mice compared with chemotherapy alone (Figure 5F); the survival of mice receiving bevacizumab only after chemotherapy (MST 56 days, ILS 124\%) was not different from mice treated with bevacizumab concomitantly with PTX + DDP and continued (MST 59 days, ILS 136\%) (detailed results in Supplementary data, Table 4).

1A9-luc is a tumour that produces solid tumour masses, but limited ascites. Therefore, a similar study was conducted on 1A9-VS1, its variant producing high levels of VEGF and ascites 
(Manenti et al, 2005). Although less efficient on the whole, bevacizumab added in the therapy significantly prolonged survival, but in this model too survival of mice treated with bevacizumab concomitantly with PTX + DDP and continued, was not better than mice given bevacizumab only after chemotherapy (Supplementary Data, Table 5).

\section{DISCUSSION}

The antitumour activity of bevacizumab on advanced EOC as monotherapy and combined with standard-of-care chemotherapy has been shown (Burger et al, 2007; Cannistra et al, 2007; Bookman, 2009; Penson et al, 2010; O'Malley et al, 2011). Two multicenter phase III studies, GOG218 and ICON7, testing bevacizumab as front-line therapy combined with carboplatin and PTX found improvement of PFS (primary end point) in the arm with maintenance bevacizumab (Burger et al, 2011; Perren et al, 2011). Although we are awaiting the publication of the final data from those clinical studies, our preclinical study in two mouse xenograft models of human EOC indicated not only significant antitumour activity when bevacizumab was added to PTX + DDP, but also prolonged survival and some complete regressions when the treatment was maintained after suspension of chemotherapy. Altogether, our data show a trend to recapitulate clinical findings, though the lack of effect of bevacizumab on OS that is emerging from clinical trials is becoming a critical issue that needs more critical analysis (Schmidt, 2011).

The use of preclinical models enabled us to study the outcome of bevacizumab in combination with chemotherapy on EOC progression (first end point) and survival (second end point).

Antiangiogenic agents, such as bevacizumab, can be administered over long periods with manageable toxicity (Gerber and Ferrara, 2005; Shah et al, 2011). Therefore, it is feasible to use antiangiogenic therapy as a maintenance regimen and it can be expected to prevent or delay cancer recurrence. In an extensive study on the effect of anti-VEGF treatment on preclinical models, the long-term continuous exposure to anti-VEGF resulted in a longer-duration therapy and extended survival (Bagri et al, 2010). In our study, bevacizumab continued after chemotherapy significantly delayed tumour progression in the peritoneal cavity (imaging studies) and prolonged survival. Furthermore, in the HOC22 model, bevacizumab treatment maintained after PTX + DDP was stopped, led to complete tumour responses.

It is believed that treatments affecting angiogenesis, including bevacizumab, enhance the effect of chemotherapy, by normalising the tumour vasculature, lowering interstitial pressures, and improving the delivery of cytotoxic agents (Jain, 2005). At the doses we used bevacizumab inhibited angiogenesis and affected tumour vascularisation (data not shown). Therefore, vascular 'normalisation' might explain why bevacizumab in combination with chemotherapy was effective in this study. However, bevacizumab was more efficacious when combined with PTX than DDP, a drug marginally active in our EOC models, so the final outcome of the combination also depends on the tumour sensitivity to chemotherapy. Another possibility is that bevacizumab acts synergistically with PTX, which itself is a potent inhibitor of angiogenesis (Belotti et al, 1996; Bocci et al, 2002).

Although the tumour profile and experimental conditions responsible for the response to anti-VEGF-targeted agents in combination with chemotherapy are far from clear, our preclinical findings confirm the antitumour activity of bevacizumab combined with standard chemotherapy on EOC and maximal benefit with the maintenance regimen.

Bevacizumab has been reported to control ascites production in mice (Hu et al, 2002), an attractive property for patients with advanced ovarian cancer. The HOC22 model, derived from a patient's ascites that disseminates in the peritoneal cavity of nude mice and produces a large amount of ascites, is an ideal model for studying the effects of chemotherapy and bevacizumab on these aspects (Massazza et al, 1989). Bevacizumab significantly inhibited ascites (interim analysis) leading to increased survival, that was further prolonged with a maintenance regimen. However, the tumours eventually progressed and mice died with a heavy tumour burden disseminating into the peritoneal organs. On the other hand, the interim analysis showed that PTX + DDP reduced the tumour burden in the peritoneal organs. Thus, in this model the effect of the anti-VEGF treatment plus chemotherapy appears to rely heavily on the effects on ascites production and tumour growth, respectively. Interestingly, the levels of plasma VEGF that matched the tumour burden confirm its use as indicator of tumour burden rather than a biomarker of bevacizumab activity.

The effect of VEGF-targeted agents on tumour malignancy is debated (Eikesdal and Kalluri, 2009; Ellis and Hicklin, 2009). Increased invasion and metastasis have been described in certain experimental conditions (Ebos et al, 2009; Paez-Ribes et al, 2009), whereas some preclinical studies did not cast light on local tumour invasion and metastasis after bevacizumab (Ranieri et al, 2006). No clinical trial has yet provided evidence of increased malignancy on VEGF blockade, though several trials indicate that initial prolongation of PFS and improved patient response rate are not always translated into an OS (Ebos and Kerbel, 2011; Van Cutsem et al, 2011). This appears to be the case also for the clinical trials on EOC (Burger et al, 2011; Perren et al, 2011). A retrospective analysis of five placebo-controlled clinical trials that investigated the efficacy and safety of bevacizumab does not support worse clinical outcome or altered disease progression after cessation of bevacizumab therapy (Miles et al, 2011).

In the HOC22 model the necropsy of representative mice treated with bevacizumab showed reduced ascites, but massive tumour dissemination in the peritoneal cavity (at interim analysis). This was evident at killing (survival) where mice receiving bevacizumab had a higher tumour burden than those receiving chemotherapy.

Although these results need to be considered with caution, they call for careful analysis of the effects of this therapy on EOC progression. The findings suggest that bevacizumab is effective on tumour progression, but it necessitates chemotherapy to control tumour dissemination and metastasis.

The timing for VEGF targeting therapy still calls for consideration. As expected, bevacizumab was ineffective on advanced 1A9-luc tumour (starting on day 21, Figure 5). Surprisingly, however, in mice bevacizumab started after induction with PTX + DDP (confirmed by imaging analysis) or started together with chemotherapy and continued after it was stopped, was equally effective on survival. Treatment with an inhibitor of angiogenesis starting after induction with chemotherapy should be worth considering to reduce the stress and discomfort of ovarian cancer patients, while maintaining the efficacy of the therapy. Further investigation is needed to determine the optimal utilisation of chemotherapy plus bevacizumab, that would support clinical holding of Bev treatment after the conclusion of chemotherapy.

In conclusion, we found overall efficacy of bevacizumab combined with chemotherapy in these mouse EOC xenograft models. The response and outcome appear related to the different phenotype and malignant behaviour of the two models, biological aspects that deserve further investigation. The doses of bevacizumab and chemotherapy we used were well tolerated and are consistent with previous preclinical reports, and roughly equivalent to standard clinical doses. Bevacizumab is currently being used added to standard chemotherapy in women with newly diagnosed ovarian cancer. Our findings are consistent with the clinical results showing a significant increase of PFS for patients treated concurrently with carboplatin (we used DDP) and PTX plus continued bevacizumab (Burger et al, 2011; Perren et al, 2011), and shed light on the potential effects of this kind of treatment on tumour progression. 


\section{ACKNOWLEDGEMENTS}

We thank Mrs Judith Baggott who provided editing assistance. Research was supported by the Italian Association for Cancer Research and Fondazione CARIPLO (no. 2008-2264) to Raffaella Giavazzi.

\section{REFERENCES}

Bagri A, Berry L, Gunter B, Singh M, Kasman I, Damico LA, Xiang H, Schmidt M, Fuh G, Hollister B, Rosen O, Plowman GD (2010) Effects of anti-VEGF treatment duration on tumor growth, tumor regrowth, and treatment efficacy. Clin Cancer Res 16: 3887-3900

Bamberger ES, Perrett CW (2002) Angiogenesis in epithelial ovarian cancer. Mol Pathol 55: 348-359

Bani MR, Nicoletti MI, Alkharouf NW, Ghilardi C, Petersen D, Erba E, Sausville EA, Liu ET, Giavazzi R (2004) Gene expression correlating with response to paclitaxel in ovarian carcinoma xenografts. Mol Cancer Ther 3: $111-121$

Barton DP, Cai A, Wendt K, Young M, Gamero A, De Cesare S (1997) Angiogenic protein expression in advanced epithelial ovarian cancer. Clin Cancer Res 3: 1579-1586

Belotti D, Vergani V, Drudis T, Borsotti P, Pitelli MR, Viale G, Giavazzi R, Taraboletti G (1996) The microtubule-affecting drug paclitaxel has antiangiogenic activity. Clin Cancer Res 2: 1843-1849

Bocci G, Nicolaou KC, Kerbel RS (2002) Protracted low-dose effects on human endothelial cell proliferation and survival in vitro reveal a selective antiangiogenic window for various chemotherapeutic drugs. Cancer Res 62: 6938-6943

Boocock CA, Charnock-Jones DS, Sharkey AM, McLaren J, Barker PJ, Wright KA, Twentyman PR, Smith SK (1995) Expression of vascular endothelial growth factor and its receptors Flt and KDR in ovarian carcinoma. J Natl Cancer Inst 87: 506-516

Bookman MA (2009) Trials with impact on clinical management: first line. Int J Gynecol Cancer 19: S55-S62

Burger RA, Brady MF, Bookman MA, Fleming GF, Monk BJ, Huang $\mathrm{H}_{\text {, }}$ Mannel RS, Homesley HD, Fowler J, Greer BE, Boente M, Birrer MJ, Liang SX (2011) Incorporation of bevacizumab in the primary treatment of ovarian cancer. $N$ Engl J Med 365: 2473-2483

Burger RA, Sill MW, Monk BJ, Greer BE, Sorosky JI (2007) Phase II trial of bevacizumab in persistent or recurrent epithelial ovarian cancer or primary peritoneal cancer: a Gynecologic Oncology Group Study. J Clin Oncol 25: 5165-5171

Cannistra SA, Bast Jr RC, Berek JS, Bookman MA, Crum CP, DePriest PD, Garber JE, Koh WJ, Markman M, McGuire 3rd WP, Rose PG, Rowinsky EK, Rustin GJ, Skates SJ, Vasey PA, King L (2003) Progress in the management of gynecologic cancer: consensus summary statement. J Clin Oncol 21: 129s-132s

Cannistra SA, Matulonis UA, Penson RT, Hambleton J, Dupont J, Mackey H, Douglas J, Burger RA, Armstrong D, Wenham R, McGuire W (2007) Phase II study of bevacizumab in patients with platinum-resistant ovarian cancer or peritoneal serous cancer. J Clin Oncol 25: 5180-5186

Chura JC, Van Iseghem K, Downs Jr LS, Carson LF, Judson PL (2007) Bevacizumab plus cyclophosphamide in heavily pretreated patients with recurrent ovarian cancer. Gynecol Oncol 107: 326-330

Codegoni AM, Nicoletti MI, Buraggi G, Valoti G, Giavazzi R, D’Incalci M, Landoni F, Maneo A, Broggini M (1998) Molecular characterisation of a panel of human ovarian carcinoma xenografts. Eur J Cancer 34: 1432-1438

Cohen MH, Shen YL, Keegan P, Pazdur R (2009) FDA drug approval summary: bevacizumab (Avastin) as treatment of recurrent glioblastoma multiforme. Oncologist 14: 1131-1138

Dvorak HF, Sioussat TM, Brown LF, Berse B, Nagy JA, Sotrel A, Manseau EJ, Van de Water L, Senger DR (1991) Distribution of vascular permeability factor (vascular endothelial growth factor) in tumors: concentration in tumor blood vessels. J Exp Med 174: 1275-1278

Ebos JM, Kerbel RS (2011) Antiangiogenic therapy: impact on invasion, disease progression, and metastasis. Nat Rev Clin Oncol 8: 210-221

Ebos JM, Lee CR, Cruz-Munoz W, Bjarnason GA, Christensen JG, Kerbel RS (2009) Accelerated metastasis after short-term treatment with a potent inhibitor of tumor angiogenesis. Cancer Cell 15: 232-239

Eikesdal HP, Kalluri R (2009) Drug resistance associated with antiangiogenesis therapy. Semin Cancer Biol 19: 310-317

\section{Conflict of interest}

The authors declare no conflict of interest.

Supplementary Information accompanies the paper on British Journal of Cancer website (http://www.nature.com/bjc)
Ellis LM, Hicklin DJ (2009) Resistance to targeted therapies: refining anticancer therapy in the era of molecular oncology. Clin Cancer Res 15: 7471-7478

Ferrara N (2004) Vascular endothelial growth factor: basic science and clinical progress. Endocr Rev 25: 581-611

Garofalo A, Naumova E, Manenti L, Ghilardi C, Ghisleni G, Caniatti M, Colombo T, Cherrington JM, Scanziani E, Nicoletti MI, Giavazzi R (2003) The combination of the tyrosine kinase receptor inhibitor SU6668 with paclitaxel affects ascites formation and tumor spread in ovarian carcinoma xenografts growing orthotopically. Clin Cancer Res 9: 3476-3485

Gerber HP, Ferrara N (2005) Pharmacology and pharmacodynamics of bevacizumab as monotherapy or in combination with cytotoxic therapy in preclinical studies. Cancer Res 65: 671-680

Giannakakou P, Poy G, Zhan Z, Knutsen T, Blagosklonny MV, Fojo T (2000) Paclitaxel selects for mutant or pseudo-null p53 in drug resistance associated with tubulin mutations in human cancer. Oncogene 19: 3078-3085

Hu L, Hofmann J, Zaloudek C, Ferrara N, Hamilton T, Jaffe RB (2002) Vascular endothelial growth factor immunoneutralization plus Paclitaxel markedly reduces tumor burden and ascites in athymic mouse model of ovarian cancer. Am J Pathol 161: 1917-1924

Hurwitz H, Fehrenbacher L, Novotny W, Cartwright T, Hainsworth J, Heim W, Berlin J, Baron A, Griffing S, Holmgren E, Ferrara N, Fyfe G, Rogers B, Ross R, Kabbinavar F (2004) Bevacizumab plus irinotecan, fluorouracil, and leucovorin for metastatic colorectal cancer. $N$ Engl J Med 350: 2335-2342

Jain RK (2005) Normalization of tumor vasculature: an emerging concept in antiangiogenic therapy. Science 307: 58-62

Kim KJ, Li B, Houck K, Winer J, Ferrara N (1992) The vascular endothelial growth factor proteins: identification of biologically relevant regions by neutralizing monoclonal antibodies. Growth Factors 7: 53-64

Kraft A, Weindel K, Ochs A, Marth C, Zmija J, Schumacher P, Unger C, Marme D, Gastl G (1999) Vascular endothelial growth factor in the sera and effusions of patients with malignant and nonmalignant disease. Cancer 85: 178-187

Lin YS, Nguyen C, Mendoza JL, Escandon E, Fei D, Meng YG, Modi NB (1999) Preclinical pharmacokinetics, interspecies scaling, and tissue distribution of a humanized monoclonal antibody against vascular endothelial growth factor. J Pharmacol Exp Ther 288: 371-378

Mabuchi S, Terai Y, Morishige K, Tanabe-Kimura A, Sasaki H, Kanemura M, Tsunetoh S, Tanaka Y, Sakata M, Burger RA, Kimura T, Ohmichi M (2008) Maintenance treatment with bevacizumab prolongs survival in an in vivo ovarian cancer model. Clin Cancer Res 14: 7781-7789

Manenti L, Paganoni P, Floriani I, Landoni F, Torri V, Buda A, Taraboletti G, Labianca R, Belotti D, Giavazzi R (2003) Expression levels of vascular endothelial growth factor, matrix metalloproteinases 2 and 9 and tissue inhibitor of metalloproteinases 1 and 2 in the plasma of patients with ovarian carcinoma. Eur J Cancer 39: 1948-1956

Manenti L, Riccardi E, Marchini S, Naumova E, Floriani I, Garofalo A, Dossi R, Marrazzo E, Ribatti D, Scanziani E, Bani M, Belotti D, Broggini M, Giavazzi R (2005) Circulating plasma vascular endothelial growth factor in mice bearing human ovarian carcinoma xenograft correlates with tumor progression and response to therapy. Mol Cancer Ther 4: 715-725

Massazza G, Tomasoni A, Lucchini V, Allavena P, Erba E, Colombo N, Mantovani A, D’Incalci M, Mangioni C, Giavazzi R (1989) Intraperitoneal and subcutaneous xenografts of human ovarian carcinoma in nude mice and their potential in experimental therapy. Int J Cancer 44: 494-500

Miles D, Harbeck N, Escudier B, Hurwitz H, Saltz L, Van Cutsem E, Cassidy J, Mueller B, Sirzen F (2011) Disease course patterns after discontinuation of bevacizumab: pooled analysis of randomized phase III trials. J Clin Oncol 29: 83-88

Nicoletti MI, Lucchini V, Massazza G, Abbott BJ, D’Incalci M, Giavazzi R (1993) Antitumor activity of taxol (NSC-125973) in human ovarian 
carcinomas growing in the peritoneal cavity of nude mice. Ann Oncol 4: 151-155

Nimeiri HS, Oza AM, Morgan RJ, Friberg G, Kasza K, Faoro L, Salgia R, Stadler WM, Vokes EE, Fleming GF (2008) Efficacy and safety of bevacizumab plus erlotinib for patients with recurrent ovarian, primary peritoneal, and fallopian tube cancer: a trial of the Chicago, $\mathrm{PMH}$, and California Phase II Consortia. Gynecol Oncol 110: 49-55

O'Malley DM, Richardson DL, Rheaume PS, Salani R, Eisenhauer EL, McCann GA, Fowler JM, Copeland LJ, Cohn DE, Backes FJ (2011) Addition of bevacizumab to weekly paclitaxel significantly improves progression-free survival in heavily pretreated recurrent epithelial ovarian cancer. Gynecol Oncol 121: 269-272

Paez-Ribes M, Allen E, Hudock J, Takeda T, Okuyama H, Vinals F, Inoue M, Bergers G, Hanahan D, Casanovas O (2009) Antiangiogenic therapy elicits malignant progression of tumors to increased local invasion and distant metastasis. Cancer Cell 15: 220-231

Penson RT, Dizon DS, Cannistra SA, Roche MR, Krasner CN, Berlin ST, Horowitz NS, Disilvestro PA, Matulonis UA, Lee H, King MA, Campos SM (2010) Phase II study of carboplatin, paclitaxel, and bevacizumab with maintenance bevacizumab as first-line chemotherapy for advanced mullerian tumors. J Clin Oncol 28: 154-159

Perren TJ, Swart AM, Pfisterer J, Ledermann JA, Pujade-Lauraine E, Kristensen G, Carey MS, Beale P, Cervantes A, Kurzeder C, du Bois A, Sehouli J, Kimmig R, Stahle A, Collinson F, Essapen S, Gourley C, Lortholary A, Selle F, Mirza MR, Leminen A, Plante M, Stark D, Qian W, Parmar MK, Oza AM (2011) A phase 3 trial of bevacizumab in ovarian cancer. $N$ Engl J Med 365: 2484-2496

Presta LG, Chen H, O'Connor SJ, Chisholm V, Meng YG, Krummen L, Winkler M, Ferrara N (1997) Humanization of an anti-vascular endothelial growth factor monoclonal antibody for the therapy of solid tumors and other disorders. Cancer Res 57: 4593-4599

Ranieri G, Patruno R, Ruggieri E, Montemurro S, Valerio P, Ribatti D (2006) Vascular endothelial growth factor (VEGF) as a target of bevacizumab in cancer: from the biology to the clinic. Curr Med Chem 13: $1845-1857$

Rini BI, Halabi S, Rosenberg JE, Stadler WM, Vaena DA, Ou SS, Archer L, Atkins JN, Picus J, Czaykowski P, Dutcher J, Small EJ (2008) Bevacizumab plus interferon alfa compared with interferon alfa monotherapy in patients with metastatic renal cell carcinoma: CALGB 90206. J Clin Oncol 26: 5422-5428

Sandler A, Gray R, Perry MC, Brahmer J, Schiller JH, Dowlati A, Lilenbaum R, Johnson DH (2006) Paclitaxel-carboplatin alone or with bevacizumab for non-small-cell lung cancer. $N$ Engl J Med 355: 2542-2550

Santin AD, Hermonat PL, Ravaggi A, Cannon MJ, Pecorelli S, Parham GP (1999) Secretion of vascular endothelial growth factor in ovarian cancer. Eur J Gynaecol Oncol 20: 177-181

Schmidt C (2011) Ovarian cancer treatments on the horizon. J Natl Cancer Inst 103: 1284-1285

Shah DK, Veith J, Bernacki RJ, Balthasar JP (2011) Evaluation of combined bevacizumab and intraperitoneal carboplatin or paclitaxel therapy in a mouse model of ovarian cancer. Cancer Chemother Pharmacol 68: 951-958

Sher I, Adham SA, Petrik J, Coomber BL (2009) Autocrine VEGF-A/KDR loop protects epithelial ovarian carcinoma cells from anoikis. Int $J$ Cancer 124: 553-561

Siegel R, Ward E, Brawley O, Jemal A (2011) Cancer statistics, 2011: the impact of eliminating socioeconomic and racial disparities on premature cancer deaths. CA Cancer J Clin 61: 212-236

Van Cutsem E, Lambrechts D, Prenen H, Jain RK, Carmeliet P (2011) Lessons from the adjuvant bevacizumab trial on colon cancer: what next? J Clin Oncol 29: 1-4

Workman P, Aboagye EO, Balkwill F, Balmain A, Bruder G, Chaplin DJ, Double JA, Everitt J, Farningham DA, Glennie MJ, Kelland LR, Robinson V, Stratford IJ, Tozer GM, Watson S, Wedge SR, Eccles SA (2010) Guidelines for the welfare and use of animals in cancer research. $\mathrm{Br} J$ Cancer 102: 1555-1577

This work is published under the standard license to publish agreement. After 12 months the work will become freely available and the license terms will switch to a Creative Commons Attribution-NonCommercial-Share Alike 3.0 Unported License. 